Draft Version February 28, 2021

Typeset using LATEX twocolumn style in AASTeX63

\title{
Competitive Dehydrogenation and Backbone Fragmentation of Super-Hydrogenated PAHs: A Laboratory Study
}

\author{
Mark H. Stockett, ${ }^{1}$ Lorenzo Avaldi, ${ }^{2}$ Paola Bolognesi,${ }^{2}$ James N. Bull,${ }^{3}$ Laura Carlini, ${ }^{2}$ \\ Eduardo Carrascosa,${ }^{4}$ Jacopo Chiarinelli, ${ }^{2}$ Robert Richter, ${ }^{5}$ and Henning Zettergren ${ }^{1}$ \\ ${ }^{1}$ Fysikum, Stockholms Universitet, Stockholm, Sweden \\ ${ }^{2}$ CNR-Istituto di Struttura della Materia, Area della Ricerca di Roma 1, Rome, Italy \\ ${ }^{3}$ School of Chemistry, University of East Anglia, Norwich, United Kingdom \\ ${ }^{4}$ Laboratoire de Chimie Physique Moléculaire, École Polytechnique Fédérale de Lausanne, Lausanne, Switzerland \\ ${ }^{5}$ Elettra Sincrotrone Trieste, Trieste, Italy
}

\begin{abstract}
Super-hydrogenated Polycyclic Aromatic Hydrocarbons (PAHs) have been suggested to catalyze the formation of $\mathrm{H}_{2}$ in certain regions of space, but it remains unclear under which circumstances this mechanism is viable given the reduced stability of super-hydrogenated PAHs. We report a laboratory study on the stability of the smallest pericondensed $\mathrm{PAH}$, pyrene $\left(\mathrm{C}_{16} \mathrm{H}_{10+N}\right.$, with $N=4,6$, and 16 additional $\mathrm{H}$ atoms), against photodestruction by single vacuum ultraviolet photons using the PhotoElectron Photo-Ion Coincidence technique. For $N=4$, we observe a protective effect of hydrogenation against the loss of native hydrogens, in the form of an increase in the appearance energies of the $\mathrm{C}_{16} \mathrm{H}_{9}^{+}$ and $\mathrm{C}_{16} \mathrm{H}_{8}^{+}$daughter ions compared to those reported for pristine pyrene $\left(\mathrm{C}_{16} \mathrm{H}_{10}\right)$. No such effect is seen for $N=6$ or 16 , where the weakening effect of replacing aromatic bonds with aliphatic ones outweighs the buffering effect of the additional hydrogen atoms. The onset of fragmentation occurs at similar internal energies for $N=4$ and 6 , but is significantly lower for $N=16$. In all three cases, H-loss and $\mathrm{C}_{m} \mathrm{H}_{n}$-loss ( $m \geq 1$, carbon backbone fragmentation) channels open at approximately the same energy. The branching fractions of the primary channels favor $\mathrm{H}$-loss for $N=4, \mathrm{C}_{m} \mathrm{H}_{n}$-loss for $N=16$, and are roughly equal for the intermediate $N=6$. We conclude that super-hydrogenated pyrene is probably too small to support catalytic $\mathrm{H}_{2}$-formation, while trends in the current and previously reported data suggest that larger PAHs may serve as catalysts up to a certain level of hydrogenation.
\end{abstract}

\section{INTRODUCTION}

Molecular hydrogen is the most abundant molecule in the universe and the key ingredient for star formation (Tielens 2005). It has been proposed that, under certain circumstances, Polycyclic Aromatic Hydrocarbons (PAHs) may play a catalytic role in $\mathrm{H}_{2}$ formation (Bauschlicher Jr 1998; Hirama et al. 2004; Castellanos, P. et al. 2018a). This may be particularly important in regions that are too warm $(>20 \mathrm{~K})$ for the otherwise successful model, association of $\mathrm{H}$ atoms on the surface of dust grains (Hollenbach \& Salpeter 1971), to operate efficiently. For example, inexplicably high $\mathrm{H}_{2}-$ formation rates have been inferred for photodissociation regions (PDRs) with elevated PAH abundances (Habart et al. 2003, 2004). Super-hydrogenated PAHs (HPAHs), which contain extra $\mathrm{H}$ atoms in addition to the native

Corresponding author: Mark H. Stockett

Mark.Stockett@fysik.su.se ones present in pristine PAHs, may play an important role in molecular hydrogen formation in these regions (Mennella et al. 2012; Ferullo et al. 2019). While not definitively identified in space, laboratory (Wagner et al. 2000; Sandford et al. 2013) and computational (Pauzat \& Ellinger 2001; Mackie et al. 2018) studies have suggested that HPAHs may contribute to the so-called Aromatic Infrared Bands (Tielens 2008) at wavelengths between 3.4-3.6 $\mu \mathrm{m}$. Highly hydrogenated PAHs have been shown to be readily formed under a variety of circumstances (Thrower et al. 2012; Boschman et al. 2012; Klærke et al. 2013; Thrower et al. 2014; Cazaux et al. 2016; Cruz-Diaz et al. 2020; Schlathölter et al. 2020), and efficient photo-induced $\mathrm{H}_{2}$ formation from HPAHs has been demonstrated (Vala et al. 2009; Szczepanski et al. 2010; Fu et al. 2011; Foley et al. 2018).

The formation, destruction, and properties of HPAHs are topical subjects within laboratory astrophysics. A central question is the stability of HPAHs under the conditions prevailing in space. The binding of addi- 

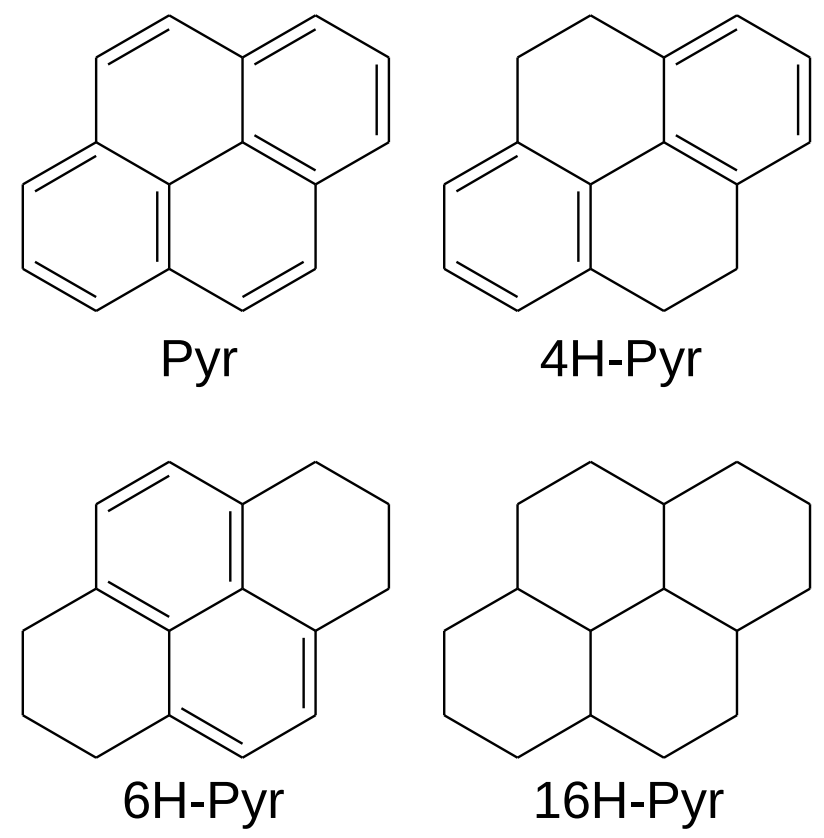

Figure 1. Pristine pyrene (Pyr, $\left.\mathrm{C}_{16} \mathrm{H}_{10}\right)$, tetrahydropyrene (4H-Pyr, $\left.\mathrm{C}_{16} \mathrm{H}_{14}\right)$, hexahydropyrene (6H-Pyr, $\mathrm{C}_{16} \mathrm{H}_{16}$ ), and hexadecahydropyrene (16H-Pyr, $\left.\mathrm{C}_{16} \mathrm{H}_{26}\right)$.

tional $\mathrm{H}$ atoms to PAHs replaces aromatic $s p^{2}$ bonds with aliphatic $s p^{3}$ bonds, weakening the carbon backbone. On the other hand, the additional $\mathrm{H}$ atoms may act as a buffer against dissociation. This was demonstrated in experiments where super-hydrogenated coronene $\left(\mathrm{C}_{24} \mathrm{H}_{12+N}, N=1,3,5\right)$ cations were found to lose fewer of their native $\mathrm{H}$ atoms with increasing levels of super-hydrogenation following soft x-ray excitation at $285 \mathrm{eV}$ (Reitsma et al. 2014). More recent studies, however, showed that higher degrees of super-hydrogenation $(N \gtrsim 6)$ led to increased carbon backbone fragmentation (Rapacioli et al. 2018; Cazaux et al. 2019). Taken together, these studies suggest the existence of a window of hydrogenation levels consistent with truly catalytic $\mathrm{H}_{2}$-formation without destruction of the native PAH. The challenge is to constrain the width of this window for a range of $\mathrm{PAH}$ sizes to ascertain under which astronomical conditions this process may be relevant.

In this article, we present a laboratory study of superhydrogenated pyrene $\mathrm{C}_{16} \mathrm{H}_{10+N}$, where $N=4,6$, and 16 additional hydrogen atoms. The structures of these molecules are presented in Figure 1. Previous investigations of this model system have shown that hydrogenation leads both to larger fragmentation cross sections (Gatchell et al. 2015) and lower threshold energies for destruction in collisions with atoms (Stockett et al. 2015; Wolf et al. 2016a). Such collisions with H, He, and C atoms are thought to be an important destruction mech- anism for PAHs in supernova shocks (Micelotta et al. 2010; Postma et al. 2010). It was further shown that less energy was required to induce fragmentation of the carbon backbone for hydrogenated pyrene than pristine pyrene in multiphoton dissociation mass spectrometry experiments (Wolf et al. 2016b). Here, we utilize the Photo-Electron Photo-Ion Coincidence (PEPICO) technique to elucidate the lowest-energy dissociation pathways of super-hydrogenated pyrene upon the absorption of single vacuum ultraviolet (VUV) photons. This is perhaps the most relevant form of activation for comparison to astrochemical models, where heating by single VUV photons is supposed to induce dissociation of PAHs in competition with vibrational infrared emission (Allamandola et al. 1989). hile both pyrene and coronene are smaller than those thought to be abundant in PDRs, they include the essential chemical groups relevant to $\mathbf{H}_{2}$-formation.

Pristine pyrene $\left(\mathrm{C}_{16} \mathrm{H}_{10}\right)$, the smallest pericondensed PAH, has been the subject of several VUVphotodissociation experiments (Ling et al. 1995; Jochims et al. 1999; West et al. 2014c; Rouillé et al. 2015) and closely related photoelectron spectroscopy studies (Mayer et al. 2011; Mishra et al. 2014). Of special interest has been the competition between the loss of molecular hydrogen and the sequential loss of hydrogen atoms. $\mathrm{H}_{2}$-loss is energetically favored but sequential $2 \mathrm{H}$-loss is entropically favored, with $\mathrm{H}_{2}$-emission becoming significant only at high excitation energies (Chen et al. 2015). Pyrene molecules with various substitutions have also been recently investigated (Rouillé et al. 2015; West et al. 2018), as have a few smaller HPAHs (Mayer et al. 2011; West et al. 2014a; Diedhiou et al. 2019).

Very recently, Diedhiou et al. (2020) investigated the photostability of super-hydrogenated catacondensed PAHs anthracene and phenanthrene in a PEPICO experiment where threshold electrons are detected. Compared to the same group's earlier study of pristine anthracene (West et al. 2014b), they observe a weak protective effect for dihydroanthracene in the form of an increased relative yield of the H-loss dissociation channel versus the main backbone fragmentation channel. For higher degrees of hydrogenation, backbone fragmentation dominates. The present study bridges the gap between the work of Diedhiou et al. (2020), as well as that of the Groningen group on coronene (Reitsma et al. 2014; Rapacioli et al. 2018; Foley et al. 2018), by considering the intermediate size, pericondensed pyrene system.

\section{EXPERIMENTAL METHODS}



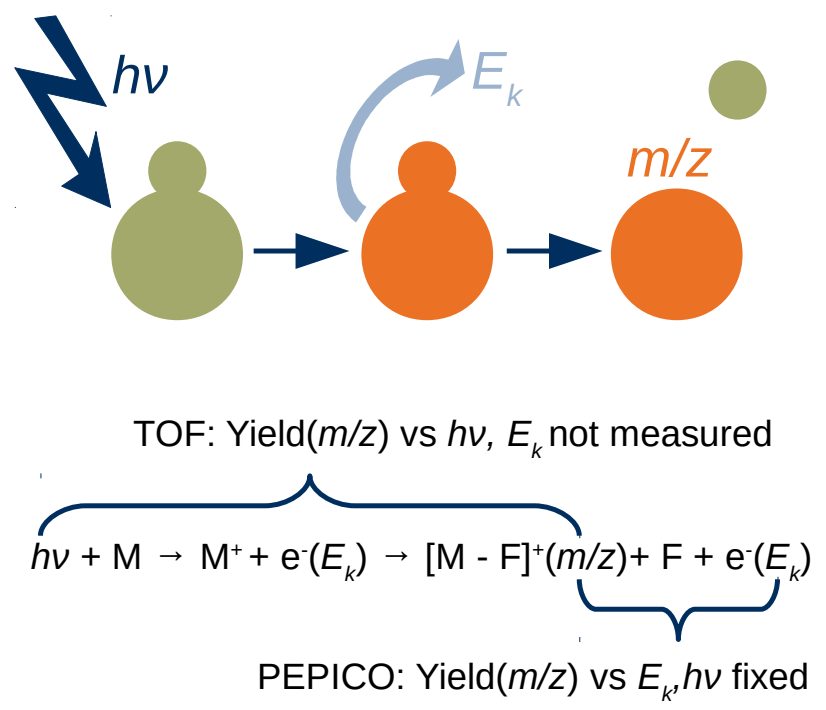

Figure 2. Comparison of the measurement principles of PEPICO and TOF methods.

Experiments were performed at the CiPO beamline (Derossi et al. 1995) of the Elettra synchrotron using an endstation designed for electron-ion coincidence experiments. The experimental setup (Plekan et al. 2008) and procedures (Chiarinelli et al. 2018) have been described previously. Briefly, the endstation is equipped with a commercial $150 \mathrm{~mm}$ mean radius hemispherical electron energy analyzer (VG 220i) mounted at the magic angle with respect to the incident linearly polarized radiation and a time-of-flight mass spectrometer mounted opposite to the electron analyzer. The two analyzers can be operated independently, for photoelectron spectroscopy (PES) and time-of-flight mass spectrometry (TOF-MS), or in time coincidence for photoelectron-photoion coincidence (PEPICO) experiments. An illustration of the different methods is given in Figure 2. Recently, the hemispherical analyzer has been equipped with a position-sensitive detector (Cautero et al. 2008; Menk et al. 2019) that replaced the original detection system made of 6 independent channeltrons (Plekan et al. 2008), leading to a significant improvement in terms of efficiency and resolution.

The PEPICO measurements were performed at a fixed photon energy of $h \nu=60 \mathrm{eV}$, with an instrumental resolution of about $0.6 \mathrm{eV}$, which mainly depends on the pass energy in the electron analyzer. In the data analysis, the yield of each fragment is determined as a function of the binding energy, after the subtraction of the random coincidences. The yield of each $m / z$ fragment has been evaluated by integrating the corresponding timeof-flight region in the PEPICO spectrum over an area of approximately $\pm 0.5 \mathrm{Da}$. The photofragment yields,

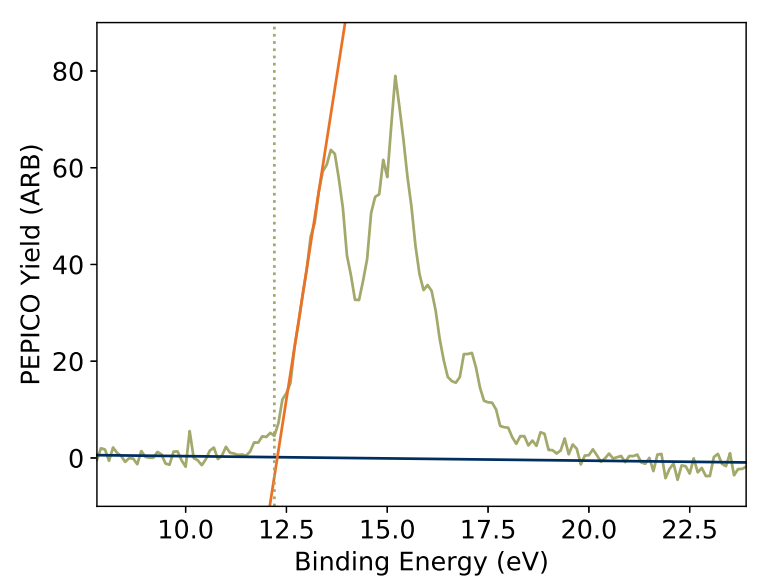

Figure 3. Example of fitting procedure for determining $A E_{P E P}$, indicated by dashed vertical line. Plotted is the PEPICO yield of the $[\mathrm{M}-\mathrm{H}]^{+}$channel of $4 \mathrm{H}-\mathrm{Pyr}$.

when given against the binding energy, represent the breakdown curves. We recorded PEPICO breakdown curves for binding energies up to about $24 \mathrm{eV}$ i.e. electron kinetic energies down to about $36 \mathrm{eV}$. By fitting the first onset of each breakdown curve with two straight lines, the crossing point $A E_{P E P}$ has been extracted and is reported in Table 1. An example of this procedure is given in Figure 3. Due to the steepness of the coincidence breakdown curves and the low statistics close to the onset, the uncertainty from these fits is rather large and the values on $A E_{P E P}$ given in Table 1 should be considered as lower bounds according to the graphical representation of the PEPICO yields. The appearance energy (AE) of a fragment is usually determined by the photoionization efficiency (PIE) measurements, i.e. measuring the ion yield versus photon/electron energy, however even though it is measured at a fixed photon energy of $60 \mathrm{eV}$, $A E_{P E P}$ can be considered as a good indication of the fragment's appearance energy.

As a complement to the PEPICO measurements, time-of-flight TOF-MS spectra were recorded over a range of photon energies $h \nu=13-32 \mathrm{eV}$ (in steps of $1 \mathrm{eV}$ for $h \nu<24 \mathrm{eV}, 2 \mathrm{eV}$ for $h \nu>24 \mathrm{eV}$ ), providing a check of the appearance energy measurements of daughter ions. In the energy range $13-16 \mathrm{eV}$ a certain amount of second-order radiation is present in the incident beam. This contribution has been evaluated and subtracted from the measured mass spectra. Compared with the analysis of the PEPICO spectra, the good statistics in the TOF measurements allowed deconvolution of the isotope distribution in the spectra by fitting each fragment group with a sum of Gaussian functions. Appearance 
energies $A E_{T O F}$ were determined by a similar procedure as $A E_{P E P}$, by fitting a straight line to the TOF branching fraction and taking the zero intercept to be $A E_{T O F}$. The uncertainties on $A E_{T O F}$ reported in Table 1 reflect only the statistical error in the fit and not the systematic errors related to the large photon energy step size or subtraction of second-order contributions.

The three samples analyzed in this work are all commercially available (TCI Europe). In all cases, an amount of sample of about $30 \mathrm{mg}$ was inserted in a crucible under vacuum. To minimize possible sample decomposition and contamination of the setup, the samples were kept at the lowest possible temperature to produce a measurable electron signal, i.e. 32 and $39{ }^{\circ} \mathrm{C}$, respectively, for $4 \mathrm{H}-$ and $6 \mathrm{H}-\mathrm{Pyr}$ and room temperature for $16 \mathrm{H}-\mathrm{Pyr}$. This produced a residual gas pressure in the range of high $10^{-8}$ - low $10^{-7} \mathrm{mbar}$, on a base pressure of the vacuum chamber of $1 \times 10^{-8}$ mbar.

\section{RESULTS AND DISCUSSION}

\subsection{Fragmentation Channels}

\subsubsection{Appearance Energies}

Figure 4 shows overview TOF mass spectra of the three target molecules following activation by $h \nu=$ $60 \mathrm{eV}$ photons. The tick marks above the peaks indicate ions observed in the (separate) PEPICO measurement. The $60 \mathrm{eV}$ TOF spectra of $4 \mathrm{H}$ - and $6 \mathrm{H}$-pyrene are qualitatively similar. Multiple peaks corresponding to H-loss channels are observed, as are ions having lost up to $4 \mathrm{C}$ atoms. Doubly charged parent molecules $\mathrm{M}^{2+}$ and daughters $\left[\mathrm{M}-\mathrm{C}_{m} \mathrm{H}_{n}\right]^{2+}$ are observed close to $m / z \approx 100$. The TOF mass spectrum of $16 \mathrm{H}$-pyrene appears different. The H-loss channels are much less prominent, while significant destruction of the carbon skeleton is observed. Compared to $4 \mathrm{H}$ - and $6 \mathrm{H}$-pyrene, many more of the low-mass daughter ions of $16 \mathrm{H}-\mathrm{Pyr}$ are observed, indicating that they open at comparatively low energy.

Table 1 gives the first onset for the photodissociation of the first several groups of fragments in $4 \mathrm{H}-, 6 \mathrm{H}-$, and 16H-Pyr, as determined from both the PEPICO measurements $\left(A E_{P E P}\right)$ and the TOF mass spectra $\left(A E_{T O F}\right)$. For the $A E_{T O F}$ determination, upper limits of $A E_{T O F}<13 \mathrm{eV}$ are given in Table 1 for cases where the channel is already open at $h \nu=13 \mathrm{eV}$, the lowest energy measured here. Upper limits are also given in cases where the TOF branching fraction could not be suitably fitted. No determinations of $A E_{T O F}$ are given for 16H-Pyr, where all channels in the table are open at $h \nu=13 \mathrm{eV}$. Note that channels are labeled by the nominal formula of the mass lost from the parent ion, with channels such as $\mathrm{CH}_{5}$-loss more properly identified as $\left[\mathrm{M}-\mathrm{CH}_{5-x}-x \mathrm{H}\right]^{+}$.

In principle, the two methods used for determining AE probe different excitation mechanisms. A PEPICO experiment, which is performed at fixed photon energy, gives photo-ion yields as a function of the internal energy of the ion. In our TOF measurements, where the yields are measured as a function of the photon energy, contributions are expected from both direct ionization and resonant excitation to bound states of the neutral decaying to the underlying ionization continuum. A comparison of the two measurement schemes is given in Figure 2. For the species considered in this work, resonant excitation is expected to lead to statistical fragmentation. That is, resonant absorption of the full photon energy $h \nu$ is assumed to be converted to internal vibrational energy and redistributed across all internal degrees of freedom before electron emission and dissociation. A significant part of this electron emission is expected to be thermionic $(\sim 0 \mathrm{eV}$ electron kinetic energy), leaving the photon energy minus the ionization energy available as internal energy for dissociation. Photofragments formed by this pathway will always appear at lower photon energies than those where significant energy is shared with the departing photoelectron, and will thus dominate the signal at the fragmentation threshold. We thus hypothesize that the PEPICO and TOF measurements should give comparable results.

Generally, the results of the two methods agree within their respective uncertainties. The few exceptions are represented by fragments of weak intensity in the PEPICO spectra which are adjacent to very intense ones in the region $m / z>200$, where the peaks are not fully resolved. In these cases, indicated in Table 1, the integration in the time-of-flight region of interest of the PEPICO spectrum includes a contribution from the tail of the neighboring fragments. In these cases, the $A E_{T O F}$ value, even though affected by a large uncertainty because of the large step in the photon energy, is more reliable as it accounts for the deconvolution of the isotope distribution.

Looking across the table, one sees that the appearance energies for "primary" fragmentation channels (e.g. [M-

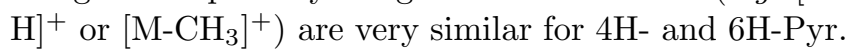
The values for multi-step channels (e.g. $\left[\mathrm{M}_{-} \mathrm{CH}_{5}\right]^{+}$i.e. $\left.\left[\mathrm{M}-\mathrm{CH}_{3}-2 \mathrm{H}\right]^{+}\right)$are somewhat lower for $6 \mathrm{H}-\mathrm{Pyr}$ than $4 \mathrm{H}-$ Pyr, by about $1 \mathrm{eV}$ on average. On the other hand, the appearance energies for daughter ions of the same mass-to-charge ratio (e.g. $m / z=205$ or 191) are mostly higher for $6 \mathrm{H}-\mathrm{Pyr}$, by more than $2 \mathrm{eV}$ on average. 16H-Pyr has lower appearance energies for all channels. While the values in Table 1 are affected 


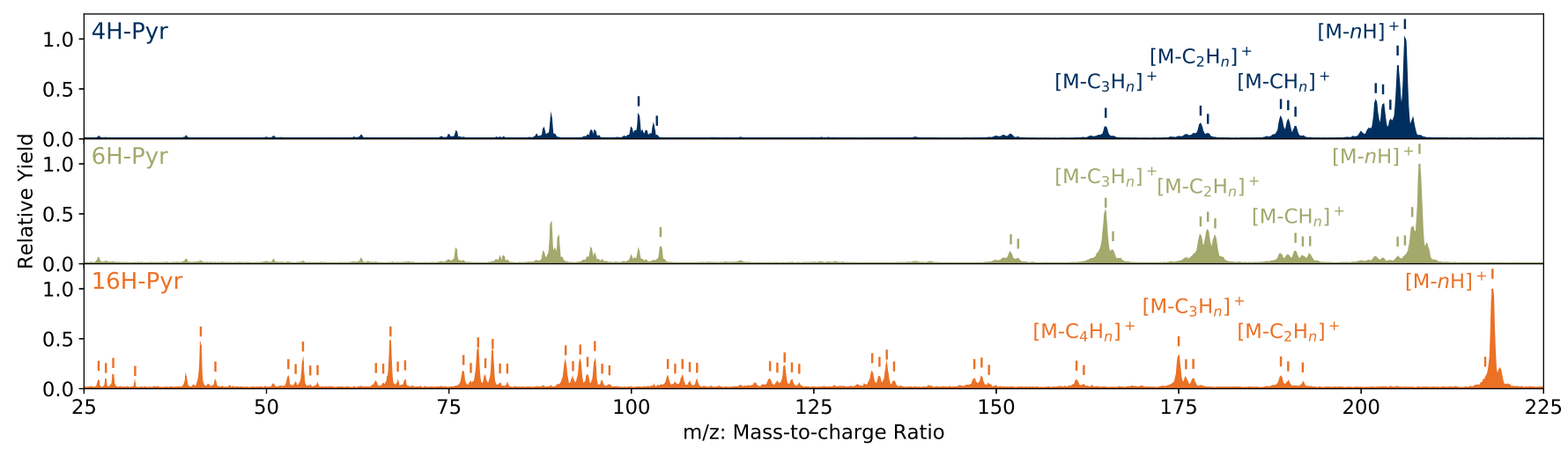

Figure 4. Overview mass spectra of $4 \mathrm{H}-, 6 \mathrm{H}-$, and $16 \mathrm{H}-\mathrm{Pyr}$ measured at $60 \mathrm{eV}$ photon energy. The marked peaks are observed in the PEPICO measurement.

Table 1. Appearance energies (in eV) for photo-ions measured using PEPICO and TOF methods. * Significant disagreements between the two methods (due to peak blending in the PEPICO data)

${ }^{\dagger}$ Minor channel, possible impurity

\begin{tabular}{|c|c|c|c|c|c|c|c|c|c|c|c|c|c|}
\hline \multirow[b]{2}{*}{ Channel } & \multicolumn{5}{|c|}{$4 \mathrm{H}-\mathrm{Pyr}$} & \multicolumn{5}{|c|}{$6 \mathrm{H}-\mathrm{Pyr}$} & \multicolumn{3}{|c|}{$16 \mathrm{H}-\mathrm{Pyr}$} \\
\hline & $m / z$ & $A E_{P E P}$ & Err. & $A E_{T O F}$ & Err. & $m / z$ & $A E_{P E P}$ & Err. & $A E_{T O F}$ & Err. & $m / z$ & $A E_{P E P}$ & Err. \\
\hline $\mathrm{M}^{+}$ & 206 & 7.1 & 0.1 & $<13$ & & 208 & 6.9 & 0.2 & $<13$ & & 218 & 8.5 & 0.1 \\
\hline$-\mathrm{H}$ & 205 & 12.2 & 0.1 & $<13$ & & 207 & 12.3 & 0.1 & $<13$ & & 217 & 10.7 & 0.1 \\
\hline$-2 \mathrm{H}$ & 204 & $15.1^{*}$ & 0.2 & $<18^{*}$ & & & & & & & & & \\
\hline$-3 \mathrm{H}$ & 203 & 14.5 & 0.1 & 14.3 & 0.3 & 205 & $14.4^{*}$ & 0.2 & $16.4^{*}$ & 0.2 & & & \\
\hline$-4 \mathrm{H}$ & 202 & $16.9^{*}$ & 0.1 & $18.1^{*}$ & 0.2 & & & & & & & & \\
\hline$-5 \mathrm{H}$ & 201 & & & 26.8 & 0.7 & & & & & & & & \\
\hline$-6 \mathrm{H}$ & 200 & & & 30.6 & 0.3 & & & & & & & & \\
\hline$-\mathrm{CH}_{3}$ & 191 & 12.7 & 0.1 & $<13$ & & 193 & 12.7 & 0.1 & $<13$ & & & & \\
\hline$-\mathrm{CH}_{4}$ & 190 & 16.3 & 0.1 & 15.9 & 0.4 & 192 & 15.8 & 0.2 & 15.3 & 0.4 & & & \\
\hline$-\mathrm{CH}_{5}$ & 189 & 18.5 & 0.1 & 18.5 & 0.1 & 191 & 17.2 & 0.1 & 17.5 & 0.1 & & & \\
\hline$-\mathrm{CH}_{6}$ & 188 & & & $<24$ & & 190 & & & 20.0 & 0.3 & & & \\
\hline$-\mathrm{CH}_{7}$ & & & & & & 189 & & & 24.7 & 0.3 & & & \\
\hline$-\mathrm{C}_{2} \mathrm{H}_{2}$ & & & & & & & & & & & $192^{\dagger}$ & 8.7 & 0.1 \\
\hline$-\mathrm{C}_{2} \mathrm{H}_{3}$ & 179 & 12.1 & 0.2 & & & & & & & & & & \\
\hline$-\mathrm{C}_{2} \mathrm{H}_{4}$ & 178 & 12.4 & 0.3 & $<13$ & & 180 & 11.5 & 0.2 & $<13$ & & 190 & 11.3 & 0.1 \\
\hline$-\mathrm{C}_{2} \mathrm{H}_{5}$ & 177 & & & 14.7 & 0.8 & 179 & 13.5 & 0.1 & 14.0 & 0.2 & 189 & 11.5 & 0.1 \\
\hline$-\mathrm{C}_{2} \mathrm{H}_{6}$ & 176 & & & 21.1 & 0.5 & 178 & 16.4 & 0.1 & 15.6 & 0.1 & & & \\
\hline$-\mathrm{C}_{2} \mathrm{H}_{7}$ & & & & & & 177 & & & 18.2 & 0.3 & & & \\
\hline$-\mathrm{C}_{3} \mathrm{H}_{5}$ & 165 & 12.5 & 0.2 & $<13$ & & & & & & & 177 & 11.4 & 0.1 \\
\hline$-\mathrm{C}_{3} \mathrm{H}_{6}$ & 164 & & & 16.0 & 1.0 & 166 & 13.2 & 0.1 & & & 176 & 11.4 & 0.1 \\
\hline$-\mathrm{C}_{3} \mathrm{H}_{7}$ & 163 & & & 26.0 & 0.6 & 165 & 13.0 & 0.2 & $<13$ & & 175 & 11.6 & 0.1 \\
\hline$-\mathrm{C}_{3} \mathrm{H}_{8}$ & & & & & & 164 & & & $<13$ & & & & \\
\hline$-\mathrm{C}_{4} \mathrm{H}_{7}$ & & & & & & 153 & 15.5 & 0.2 & & & & & \\
\hline$-\mathrm{C}_{4} \mathrm{H}_{8}$ & & & & & & & & & & & 162 & 11.6 & 0.1 \\
\hline$-\mathrm{C}_{4} \mathrm{H}_{9}$ & & & & & & & & & & & 161 & 11.4 & 0.2 \\
\hline
\end{tabular}


Table 2. Calculated dissociation energies (B3LYP/6$31 \mathrm{G}(\mathrm{d})$, in eV) for important fragmentation channels. Values for $6 \mathrm{H}$ - and $16 \mathrm{H}-\mathrm{Pyr}$ from Gatchell et al. (2015). For $\mathrm{C}_{2} \mathrm{H}_{4}{ }^{-}$ loss from $4 \mathrm{H}-\mathrm{Pyr}$, two values are given; the smaller is for direct cleavage and the larger includes re-arrangement of the remaining two additional $\mathrm{H}$ atoms.

\begin{tabular}{l|lll} 
Channel & $4 \mathrm{H}-\mathrm{Pyr}$ & $6 \mathrm{H}-\mathrm{Pyr}$ & 16H-Pyr \\
\hline$-\mathrm{H}$ & 2.4 & 2.6 & 2.0 \\
$-2 \mathrm{H}$ & 4.9 & 5.2 & 5.1 \\
$-\mathrm{CH}_{3}$ & 2.2 & 2.3 & 1.6 \\
$-\mathrm{C}_{2} \mathrm{H}_{4}$ & $1.3 / 6.1$ & 3.9 & 2.4 \\
$-\mathrm{C}_{3} \mathrm{H}_{6}$ & - & 6.2 & 2.2
\end{tabular}

by kinetic shifts, we expect such shifts to be of similar magnitude for analogous fragmentation channels. For a given dissociation rate, the kinetic shift (and thus the measured AE) should increase with the degree of hydrogenation, contrary to some of the trends identified here.

\subsubsection{Dissociation Energies}

Dissociation energies have been calculated for $4 \mathrm{H}-\mathrm{Pyr}$ by Density Functional Theory at the B3LYP/6-31G(d) level of theory using GAUSSIAN09 (Frisch et al. 2009). The calculated dissociation energies are given in Table 2 , and the structures of the parent and product ions are pictured in the Supplementary Material. Corresponding values for $6 \mathrm{H}-$ and $16 \mathrm{H}-\mathrm{Pyr}$ are taken from Gatchell et al. (2015), who used the same computational approach. Dissociation energies account only for the difference in energy between the initial and final states, and do not consider energy barriers or transition states. Thus, the dissociation energies (Table 2) are not directly comparable to the appearance energies (Table 1), and we limit our discussion here to general trends and gross discrepancies in the data.

For $4 \mathrm{H}$ - and $6 \mathrm{H}-\mathrm{Pyr}$, the experimental appearance energies for $\mathbf{H}$ - and $\mathbf{C H}_{3}$-loss (Table 1) lie about $5 \mathrm{eV}$ above the ionization threshold, or equivalently $2-3 \mathrm{eV}$ above the dissociation energy (Table 2). This excess energy - which is much larger than the energy barriers to H-loss (Cazaux et al. 2016) or H-migration (Castellanos, P. et al. 2018b) - is attributed to a kinetic shift (Lifshitz 2002). Kinetic shifts are a consequence of the rapidly increasing dissociation rate constant above threshold and the short sampling time between activation of the molecules and extraction of the photo-ions by the mass spectrometer, estimated to be $\approx 0.5 \mu$ s for our instrument. The kinetic shift for $4 \mathrm{H}-$ and $6 \mathrm{H}-\mathrm{Pyr}$ is comparable to, though somewhat less than that measured for pristine pyrene (Ling et al. 1995), although precise comparisons cannot be made between shifts measured with different instruments. In contrast, despite the larger number of vibrational degrees of freedom over which the excitation is distributed, the kinetic shift for 16H-Pyr is small, implying much higher dissociation rate constants. The similarity of the dissociation energies for H-loss and backbone fragmentation underscores the importance of the competition between these pathways to the photostability of HPAHs.

According to our calculations, the energy required to liberate a hydrogen atom is about $2.5 \mathrm{eV}$, in agreement with calculations for super-hydrogenated coronene (Cazaux et al. 2016), although higher than for some smaller HPAHs (Diedhiou et al. 2020). This value is also consistent with the experimental finding that the appearance energy for the $m / z=201,200$ daugher ions from $4 \mathrm{H}-\mathrm{Pyr}$ are shifted by about $10 \mathrm{eV}, 2.5 \mathrm{eV}$ per additional $\mathrm{H}$, relative to pristine pyrene (Ling et al. 1995; Jochims et al. 1999; West et al. 2014c; Rouillé et al. 2015).

\subsection{Branching Fractions}

\subsubsection{H-Pyr}

Figure 5 shows the relative PEPICO yields for the main fragmentation channels of $4 \mathrm{H}-\mathrm{Pyr}$. Several fragmentation channels open at similar binding energies around $12.4 \mathrm{eV}$. The loss of a single $\mathrm{H}$ atom is the channel with by far the highest branching fraction. Backbone fragmentation channels, most importantly $\mathrm{CH}_{3^{-}}$, $\mathrm{C}_{2} \mathrm{H}_{4}$-loss, are also observed. In Table 2, two values for the $\mathrm{C}_{2} \mathrm{H}_{4}$-loss dissociation energy are given. The higher value $(6.1 \mathrm{eV})$ corresponds to direct cleavage while the lower $(1.3 \mathrm{eV})$ includes the stabilizing effect of the remaining two excess $\mathrm{H}$ atoms saturating the dangling bonds at the cleavage site, giving the phenanthrene cation as the final product. The structures of these two prodcut ins are shown in Figure 6. Given that the relative yield of $\mathrm{C}_{2} \mathrm{H}_{4}$-loss is rather low, the complex rearrangement required for the low-energy pathway may be kinetically disfavored (recall that the dissociation energies do not include barriers). On the other hand, other minor channels opening at the same binding energy are $\mathrm{C}_{2} \mathrm{H}_{3}$ - and $\mathrm{C}_{3} \mathrm{H}_{5}$-loss, which have calculated dissociation energies of 3.4 and $2.3 \mathrm{eV}$, respectively. These channels do not correspond to any simple bond cleavage and suggest that H-migration is an important step in the fragmentation process (Solano \& Mayer 2015).

At higher binding energies, a sequence of further hydrogen losses emerges, as do additional backbone fragmentation channels. The $\mathrm{CH}_{4}$ - and $\mathrm{CH}_{5}$-loss channels appear at energies where the $[\mathrm{M}-\mathrm{H}]^{+}$and $[\mathrm{M}-2 \mathrm{H}]^{+}$yields 


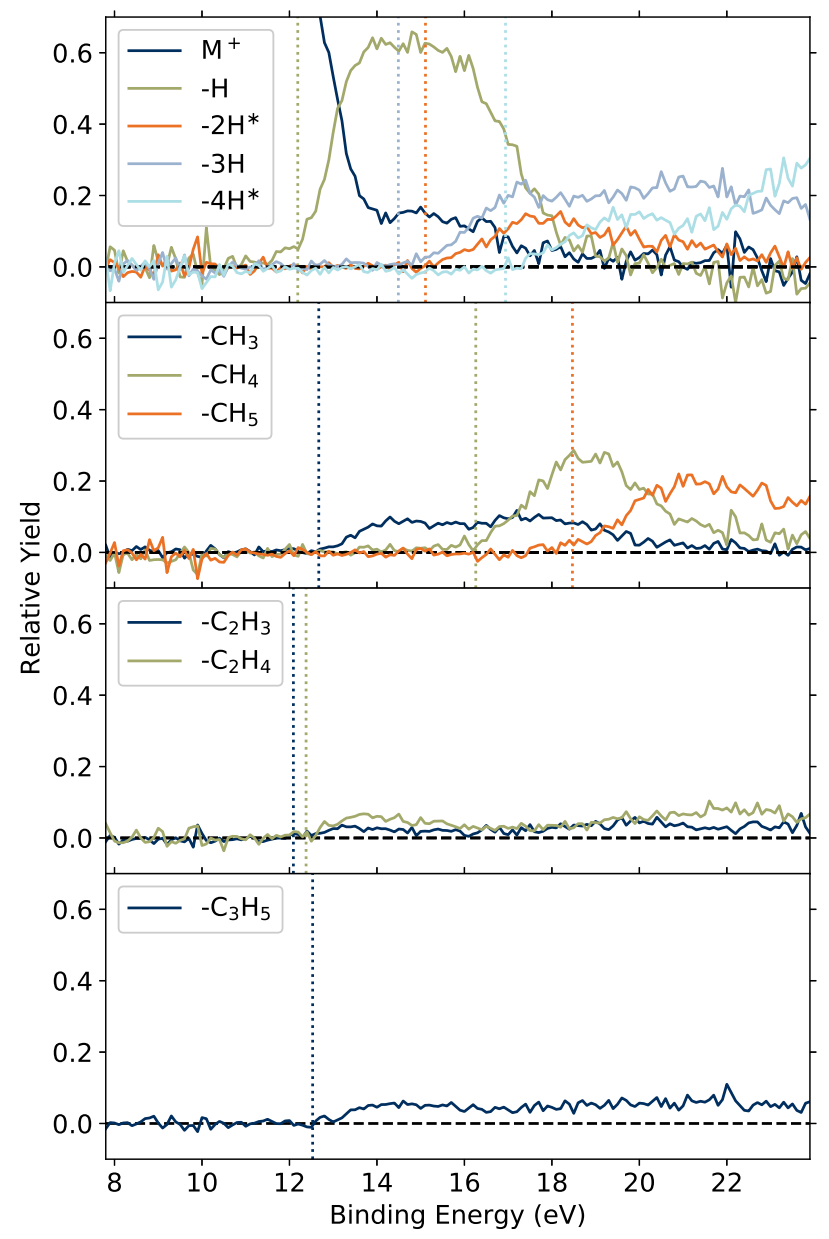

Figure 5. PEPICO yields for $n \mathrm{H}-$ and $\mathrm{C}_{m} \mathrm{H}_{n}$-loss from $4 \mathrm{H}-\mathrm{Pyr}$. Vertical dotted lines indicate $A E_{P E P}$. ${ }^{*}$ See the discussion of possible peak-blending in Section 3.1.1.
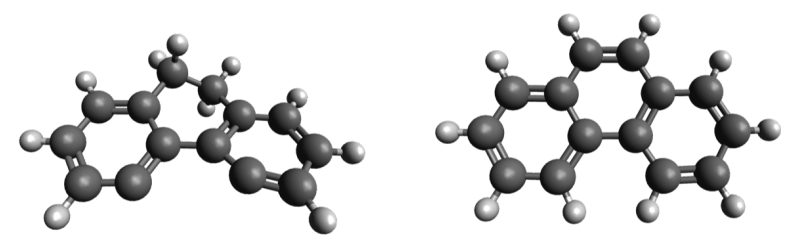

Figure 6. Structures of product ions of $\mathrm{C}_{2} \mathrm{H}_{4}$-loss from $4 \mathrm{H}$-Pyr. Left: result of direct cleavage of a $\mathrm{C}_{2} \mathrm{H}_{4}$ group with dissociation energy $6.1 \mathrm{eV}$. Right: result after rearrangement of $\mathrm{H}$ atoms yielding phenanthrene cation with dissociation energy $1.3 \mathrm{eV}$.

are decreasing, leading to the interpretation that the former ions are formed through $\mathrm{CH}_{3}$-loss from the latter i.e. $\mathrm{M}^{+} \rightarrow[\mathrm{M}-\mathrm{H}]^{+}+\mathrm{H} \rightarrow\left[\mathrm{M}-\mathrm{CH}_{4}\right]^{+}+\mathrm{H}+\mathrm{CH}_{3}$. In contrast, the $[\mathrm{M}-3 \mathrm{H}]^{+}$and $[\mathrm{M}-4 \mathrm{H}]^{+}$ions, which correspond to the protonated and radical pyrene cation, are present up to at least $24 \mathrm{eV}$.

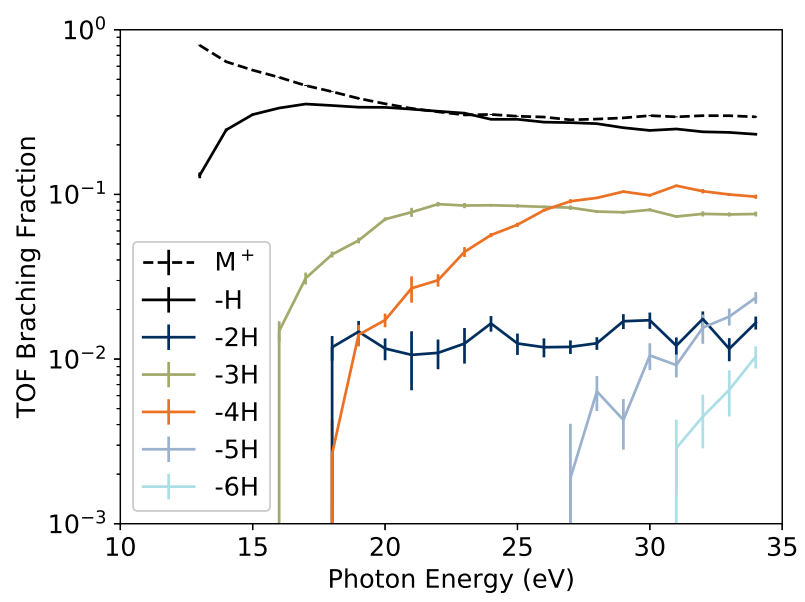

Figure 7. Relative TOF yields for $n \mathrm{H}-$ loss from $4 \mathrm{H}-\mathrm{Pyr}$.

Although not seen in the PEPICO data, the $[\mathrm{M}-5 \mathrm{H}]^{+}$ and $[\mathrm{M}-6 \mathrm{H}]^{+}$daughter ions are observed in our energydependent TOF mass spectra (which extend to higher energy), shown in Figure 7. These channels, appearing at $h \nu=26.8$ and $30.6 \mathrm{eV}$, respectively, correspond to the loss of native $\mathrm{H}$ atoms. The previously reported appearance energies for these same daughter ions starting from pristine pyrene are $16.3 \mathrm{eV}$ (Ling et al. 1995; Jochims et al. 1999; Rouillé et al. 2015) and $19.2 \mathrm{eV}$ (Ling et al. 1995). The four additional $\mathrm{H}$ atoms on $4 \mathrm{H}-$ Pyr thus provide a buffering capacity against the loss of native hydrogens on the order of $10 \mathrm{eV}$. This is an intuitive quantification of the protective effect first reported for super-hydrogenated coronene cations (Reitsma et al. 2014). We stress, however, that in the case of $4 \mathrm{H}-\mathrm{Pyr}$ there are several open backbone fragmentation pathways that compete with dehydrogenation.

\subsection{2. $6 H-P y r$}

Figure 8 shows the relative PEPICO yields for the main fragmentation channels of $6 \mathrm{H}-\mathrm{Pyr}$. As for $4 \mathrm{H}-\mathrm{Pyr}$, several fragmentation channels open around $12.4 \mathrm{eV}$. In contrast, however, the branching fractions for $\mathrm{H}-$ and $\mathrm{C}_{m} \mathrm{H}_{n}$-loss for $6 \mathrm{H}-\mathrm{Pyr}$ are nearly equal. Further dehydrogenation leading to pristine pyrene is not significant. The protective effect of super-hydrogenation is not as important for this molecule, in agreement with previous studies using other means of activation (Gatchell et al. 2015; Wolf et al. 2016a,b).

The nominal $\mathrm{C}_{3} \mathrm{H}_{7}$-loss channel is surprisingly strong. This channel likely results from cascade processes that involve either $\mathrm{C}_{2} \mathrm{H}_{4}$ - and $\mathrm{CH}_{3}$-loss or $\mathrm{C}_{3} \mathrm{H}_{6}$ - and $\mathrm{H}$-loss.

\subsection{3. $16 H-P y r$}

Figure 9 shows the relative PEPICO yields for the first several fragment groups of $16 \mathrm{H}-\mathrm{Pyr}$. Compared to 


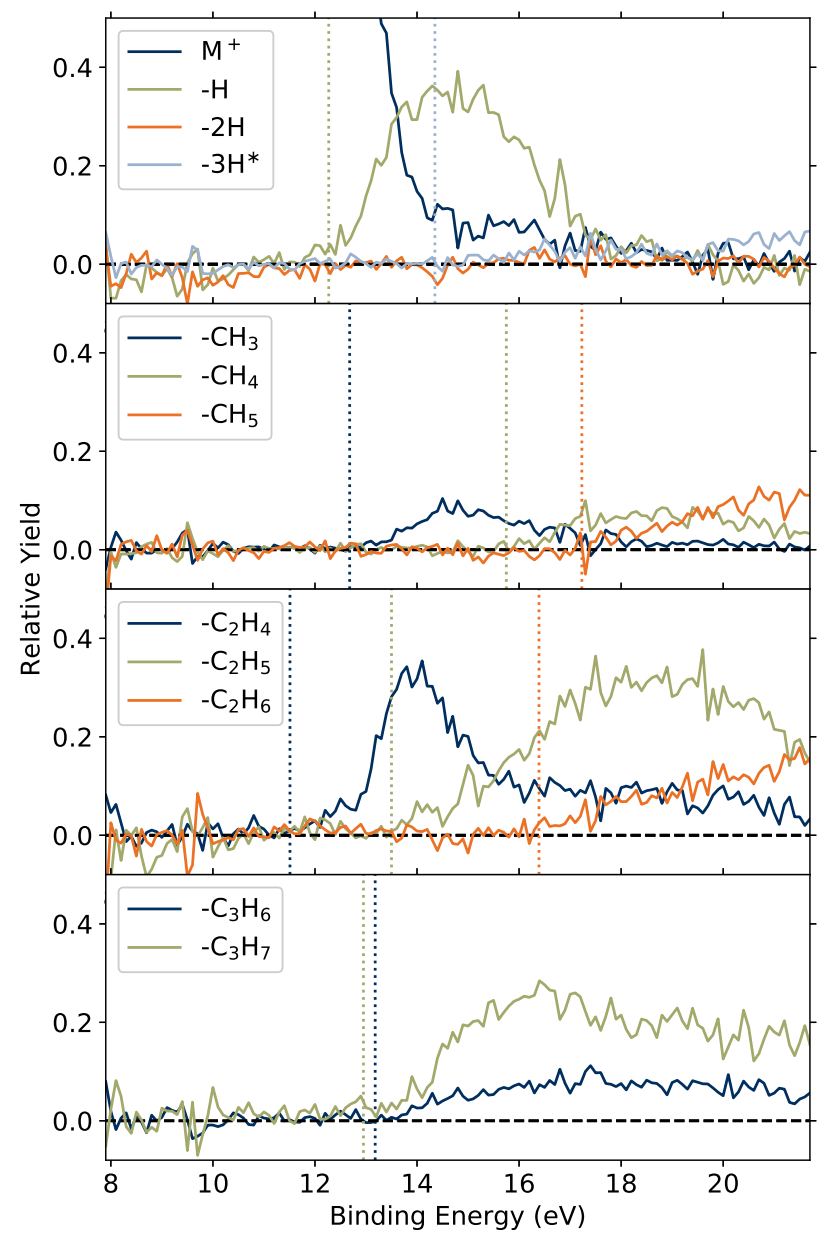

Figure 8. PEPICO yields for $n \mathrm{H}-$ and $\mathrm{C}_{m} \mathrm{H}_{n}$-loss from $6 \mathrm{H}-\mathrm{Pyr}$. Vertical dotted lines indicate $A E_{P E P}$. ${ }^{*}$ See the discussion of possible peak-blending in Section 3.1.1

$4 \mathrm{H}$ - and $6 \mathrm{H}-\mathrm{Pyr}, \mathrm{H}-\mathrm{loss}$ is a minor channel for $16 \mathrm{H}-\mathrm{Pyr}$, with backbone fragmentation dominating. Several backbone fragmentation channels and H-loss open at binding energies around $11.5 \mathrm{eV}$, only about $3 \mathrm{eV}$ above the ionization threshold.

Interestingly, $\mathrm{CH}_{3}$-loss is not detected, despite this being the channel with the lowest calculated dissociation energy (Gatchell et al. 2015). It may be that [M$\left.\mathrm{CH}_{3}\right]^{+}$daughter ions are formed but with sufficient internal energy to further fragment. Much like for $6 \mathrm{H}-$ Pyr, this could explain the prominence of channels such as $\mathrm{C}_{3} \mathrm{H}_{7}$-loss ( $\left.\left[\mathrm{M}-\mathrm{CH}_{3}-\mathrm{C}_{2} \mathrm{H}_{4}\right]^{+}\right)$and $\mathrm{C}_{4} \mathrm{H}_{9}$-loss ([M- $\mathrm{CH}_{3}$ $\left.\mathrm{C}_{3} \mathrm{H}_{6}\right]^{+}$), although these could also correspond to [M-H$\left.\mathrm{C}_{3} \mathrm{H}_{6}\right]^{+}$and $\left[\mathrm{M}-\mathrm{H}-\mathrm{C}_{4} \mathrm{H}_{8}\right]^{+}$.

Just beyond the ionization threshold for $16 \mathrm{H}-\mathrm{Pyr}$, a product with $m / z=192$ is observed, labeled as $\mathrm{C}_{2} \mathrm{H}_{2}$ loss in Figure 9. This is not an energetically favored dissociation channel, and the very low activation energy suggests that dissociation occurs along an excited

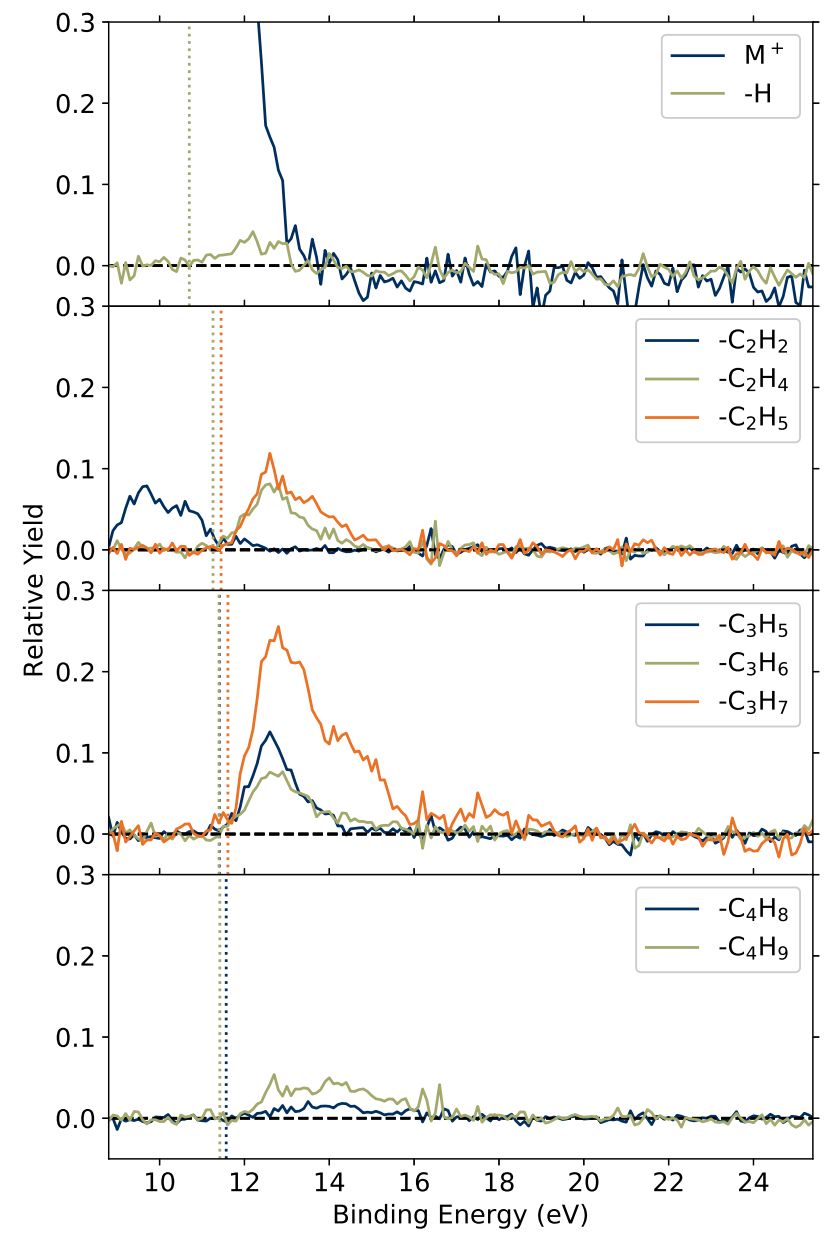

Figure 9. PEPICO yields for $\mathrm{H}$ - and $\mathrm{C}_{m} \mathrm{H}_{n}$-loss from $16 \mathrm{H}$ Pyr. Vertical dotted lines indicate $A E_{P E P}$. Note that there are several additional fragmentation channels open in this energy range (see Figure 4) which are not plotted. The total relative yield of the channels shown here is thus less than unity.

state pathway before internal conversion. However, in the present experiment, we are unable to exclude the possibility of a sample impurity such as possibly tetradecahydro-anthracene.

Regardless of the details of the fragmentation pathways, it is clear that in the extreme case of fully saturated $16 \mathrm{H}-\mathrm{Pyr}$, the weakening effect of replacing aromatic bonds with aliphatic ones is much more important than the buffering effect of the additional hydrogen atoms.

\subsubsection{Summary}

By way of summary, Figure 10 shows the branching fractions of the first few fragment groups from the PEPICO measurement at a common electron binding energy of $13.6 \mathrm{eV}$. This energy corresponds to the highest photon energy expected to impinge on HPAHs in 


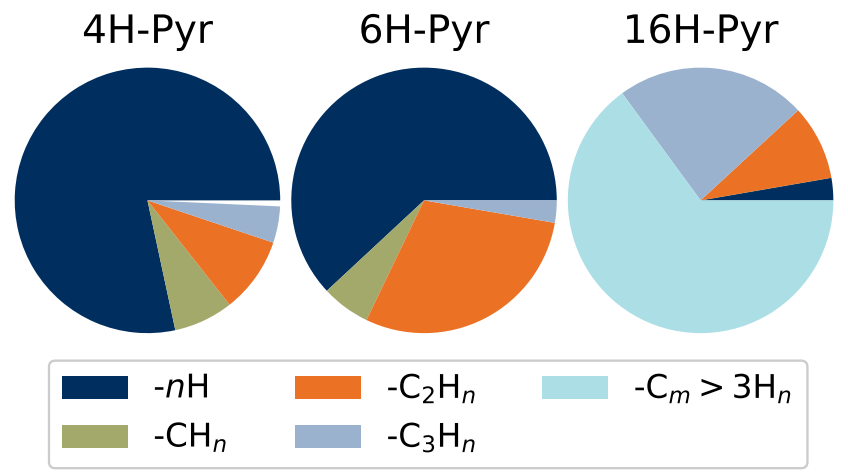

Figure 10. Comparison of the branching fractions of the first few fragment groups at a binding energy of $13.6 \mathrm{eV}$.

PDRs (Montillaud, J. et al. 2013; Boschman, L. et al. 2015). At this energy, the majority of photo-activated $4 \mathrm{H}-$ and $6 \mathrm{H}-\mathrm{Pyr}$ ions remain intact or have suffered only $\mathrm{H}$-loss. The backbone fragmentation fraction of $6 \mathrm{H}-\mathrm{Pyr}$ is more than $15 \%$ higher than that of $4 \mathrm{H}-\mathrm{Pyr}$. However, only a tiny fraction of $16 \mathrm{H}-\mathrm{Pyr}$ photoions remain intact, with most losing more than three $\mathrm{C}$ atoms. A similar trend is observed for the smaller catacondensed PAHs anthracene and phenanthrene, where $\mathrm{H}-$ and $\mathrm{CH}_{3}-$ loss are dominant for the di-hydrogenated species (West et al. 2014a; Diedhiou et al. 2020), but larger carboncontaining fragments become more important with increasing hydrogenation (Diedhiou et al. 2020). The branching fractions in Figure 10 can be compared to pristine pyrene, where $100 \%$ of photo-ions remain intact at $13.6 \mathrm{eV}$ binding energy (Ling et al. 1995; Jochims et al. 1999; Rouillé et al. 2015). Overall, the trend is consistent with decreasing stability of the carbon backbone with an increasing degree of super-hydrogenation.

\section{IMPLICATIONS FOR ASTROPHYSICS}

Multiple closely related $\mathbf{H}_{2}$-formation mechanisms involving PAHs have been proposed to explain the high observed $\mathrm{H}_{2}$ abundance in PDRs (Bauschlicher Jr 1998; Hirama et al. 2004; Mennella et al. 2012; Chen et al. 2015; Castellanos, P. et al. 2018a; Ferullo et al. 2019). Here we consider a cycle where $\mathbf{H}$ atoms are gradually added to a PAH, with sufficient time between additions for the molecule to relax to its global minimum energy structure and radiate away any excess internal excitation. $\mathrm{H}_{2}$-emission is finally induced by the absorption of a UV photon.

For a truly catalytic cycle for $\mathrm{H}_{2}$-formation involving PAHs, the carbon backbone must be preserved with near-unit probability. We have shown that this criterion is not met for super-hydrogenated pyrene. Figure 11

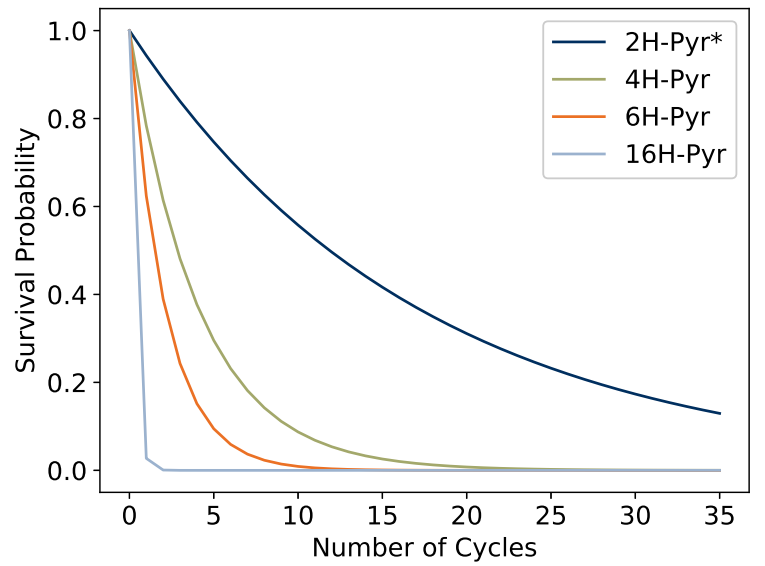

Figure 11. Depletion of HPAH "catalysts" following sequential hydrogenation-excitation-fragmentation cycles. The backbone fragmentation branching fractions (Figure 10) are taken as survival probabilities. *The survival probability of $2 \mathrm{H}-\mathrm{Pyr}$ is extrapolated from the trend from $4 \mathrm{H}-$ to $6 \mathrm{H}-\mathrm{Pyr}$.

presents a simple model for the survival probabilities upon repeated excitation assuming that the outcome follows the probability mass function of the binomial distribution. Taking the $\mathrm{C}_{m} \mathrm{H}_{n}$-loss branching fractions $(m \geq 1)$ from the previous section as destruction probabilities in competition with $\mathrm{H}_{2}$ evolution, one finds even in the best case $(4 \mathrm{H}-\mathrm{Pyr})$ that $90 \%$ of the "catalyst" is depleted after only 10 cycles, as illustrated in Figure 11. To complete a successful catalytic cycle, at least 2 added $\mathrm{H}$ atoms are required while leaving the underlying $\mathrm{PAH}$ intact. Extrapolating the trend in survival probability for $6 \mathrm{H}-$ and $4 \mathrm{H}-\mathrm{Pyr}$ down to $2 \mathrm{H}-\mathrm{Pyr}$, one may hypothesize that this minimal catalytic unit could last up to 40 cycles before $90 \%$ depletion. Note that our simple model makes the unrealistic assumption that surviving ions always return to their original hydrogenation level before the next excitation event, and the curves in Figure 11 should be viewed as average values for typical hydrogenation levels.

The stability trend of HPAHs depends on size. For the pyrene family, H-loss and backbone fragmentation have roughly equal branching fractions near threshold at a super-hydrogenation level $N=6$ (Figure 8). For the smaller catacondensed PAHs anthracene and phenanthrene, the crossover occurs already between $N=2$ and 4 additional $\mathrm{H}$ atoms (Diedhiou et al. 2020). An infrared multi-photon dissociation study of super-hydrogenated coronene found the branching fractions to be comparable at $N=7$ (Cazaux et al. 2019). For coronene and the larger PAHs expected to be predominant in PDRs, it may be expected 
- though this has not been demonstrated - that hydrogenated species with the minimal amount of superhydrogenation to achieve catalytic $\mathrm{H}_{2}$ formation could approach $100 \%$ stability against loss of native $H$ atoms and backbone fragmentation. However, as sequential hydrogenation past the first added $\mathrm{H}$ atom faces greatly reduced or vanishing energy barriers (Rauls \& Hornekær 2008), this would require fine balancing between the rates of $\mathrm{H}$-addition and photo-excitation leading to $\mathrm{H}_{2}$ evolution. In a PDR, there may thus exist a thin layer in which these processes are balanced and catalytic activity of coronene is viable. Larger PAHs with wider windows of stability upon hydrogenation could be active over correspondingly thicker layers in the PDR.

The structures of the molecules in our model system (Figure 1) are highly symmetric. Studies of super-hydrogenated coronene have found that sequential hydrogenation produces highly asymmetric structures due do the fact that attachment of a hydrogen atom structurally changes the PAH locally, facilitating attachment of more $H$ atoms in the vicinity (Cazaux et al. 2016, 2019). Asymmetric hydrogenation may lead to highly hydrogenated domains with locally weaker carbon backbones compared to a symmetrically hydrogenated PAH at the same hydrogenation level. However, it may also be that, given the lower $\mathrm{H}$ atom fluxes in PDRs compared to the experiments, asymmetrically hydrogenated HPAHs could have time to relax to globally optimal, symmetric structures.

\section{CONCLUSIONS}

We have presented a study of the stability of a series of super-hydrogenated pyrene molecules against fragmentation by VUV photons. Using the PEPICO method, we have determined the formation energetics and branching fractions for the most significant $\mathrm{H}$-loss and carbon backbone fragmentation channels. In each case, we find that $\mathrm{H}$ - and $\mathrm{C}_{m} \mathrm{H}_{n}$-loss channels open at similar ener- gies. A possible exception is the minor $\mathrm{C}_{2} \mathrm{H}_{2}$-loss channel for $16 \mathrm{H}-\mathrm{Pyr}$, which appears at a binding energy just above the ionization threshold but may be due to a sample impurity. We observe a clear trend in the branching fractions, which increasingly favor backbone fragmentation with increasing hydrogenation. Similar trends have been recently reported for smaller, catacondensed HPAHs (Diedhiou et al. 2020). We conclude that pyrene is too small to act as as true catalyst for $\mathrm{H}_{2}$ formation in PDRs.

We find that, compared to pristine $\operatorname{Pyr}\left(\mathrm{C}_{16} \mathrm{H}_{10}\right)$, the internal energies required to form the daughter ions having lost the first native hydrogens $\left(\mathrm{C}_{16} \mathrm{H}_{9}^{+}\right.$and $\left.\mathrm{C}_{16} \mathrm{H}_{8}^{+}\right)$ are about $10 \mathrm{eV}$ higher for $4 \mathrm{H}-\mathrm{Pyr}\left(\mathrm{C}_{16} \mathrm{H}_{14}\right)$, or $2.5 \mathrm{eV}$ per $\mathrm{H}$ atom. This corresponds to the typical binding energies of $\mathrm{H}$ atoms to PAH cations (Cazaux et al. 2016). We also observe that the lowest fragmentation energies are about $2 \mathrm{eV}$ higher for $6 \mathrm{H}-\mathrm{Pyr}$ than for $4 \mathrm{H}-\mathrm{Pyr}$, or $1 \mathrm{eV}$ per $\mathrm{H}$ atom.

The increasing probability of PAH backbone fragmentation, and the corresponding decrease in catalytic potential, with increasing degrees of super-hydrogenation is an important effect to consider in astrochemical models. More laboratory work is needed, particularly on 'edge' cases like small pericondensed PAHs, to support such modeling efforts with quantitative branching fractions and their trends with PAH size and structure.

This work was supported by the Swedish Research Council (grant numbers 2016-03675, 2016-04181), the Carl Trygger Foundation (grant number 17:436), the Swedish Foundation for International Collaboration in Research and Higher Education (STINT, grant number PT2017-7328 awarded to JNB, EC and MHS), and the MAECI-VR Italy-Sweden project "Novel molecular tools for the exploration of the nanoworld" (VR grant number 2018-00913). This article is based upon work from COST Action CA18212 - Molecular Dynamics in the GAS phase (MD-GAS), supported by COST (European Cooperation in Science and Technology).

\section{REFERENCES}

Allamandola, L. J., Tielens, A. G. G. M., \& Barker, J. R. 1989, Astrophys. J. Supp. Ser., 71, 733, doi: 10.1086/191396

Bauschlicher Jr, C. W. 1998, Astrophys. J. Lett., 509, L125, doi: 10.1086/311782

Boschman, L., Reitsma, G., Cazaux, S., et al. 2012,

Astrophys. J. Lett., 761, L33,

doi: 10.1088/2041-8205/761/2/L33
Boschman, L., Cazaux, S., Spaans, M., Hoekstra, R., \& Schlathölter, T. 2015, Astron. Astrophys., 579, A72, doi: 10.1051/0004-6361/201323165

Castellanos, P., Candian, A., Andrews, H., \& Tielens, A. G. G. M. 2018a, A\&A, 616, A167, doi: 10.1051/0004-6361/201833221

Castellanos, P., Candian, A., Zhen, J., Linnartz, H., \& Tielens, A. G. G. M. 2018b, Astron. Astrophys., 616, A166, doi: 10.1051/0004-6361/201833220 
Cautero, G., Sergo, R., Stebel, L., et al. 2008, Nucl. Instrum. Methods Phys. Res., Sect. A, 595, 447, doi: https://doi.org/10.1016/j.nima.2008.06.046

Cazaux, S., Arribard, Y., Egorov, D., et al. 2019, Astrophys. J., 875, 27, doi: 10.3847/1538-4357/ab0e01

Cazaux, S., Boschman, L., Rougeau, N., et al. 2016, Sci. Rep., 6, 19835, doi: 10.1038/srep19835

Chen, T., Gatchell, M., Stockett, M. H., et al. 2015, J. Chem. Phys., 142, 144305, doi: 10.1063/1.4917021

Chiarinelli, J., Bolognesi, P., Domaracka, A., et al. 2018, Phys. Chem. Chem. Phys., 20, 22841, doi: 10.1039/C8CP03473G

Cruz-Diaz, G. A., Ricca, A., \& Mattioda, A. L. 2020, ACS Earth and Space Chemistry, 4, 1730, doi: 10.1021/acsearthspacechem.0c00145

Derossi, A., Lama, F., Piacentini, M., Prosperi, T., \& Zema, N. 1995, Rev. Sci. Instrum., 66, 1718, doi: 10.1063/1.1145828

Diedhiou, M., West, B. J., Bouwman, J., \& Mayer, P. M. 2019, J. Phys. Chem. A, 123, 10885, doi: 10.1021/acs.jpca.9b09511

Diedhiou, M., West, B. J., \& Mayer, P. M. 2020, Mol. Astrophys., 19, 100071, doi: https://doi.org/10.1016/j.molap.2020.100071

Ferullo, R. M., Zubieta, C. E., \& Belelli, P. G. 2019, Phys. Chem. Chem. Phys., 21, 12012, doi: 10.1039/C9CP02329A

Foley, N., Cazaux, S., Egorov, D., et al. 2018, Mon. Not. R. Astron. Soc., 479, 649, doi: 10.1093/mnras/sty1528

Frisch, M. J., Trucks, G. W., Schlegel, H. B., et al. 2009, Gaussian 09 Revision A.01

Fu, Y., Szczepanski, J., \& Polfer, N. C. 2011, Astrophys. J., 744, 61, doi: 10.1088/0004-637X/744/1/61

Gatchell, M., Stockett, M. H., de Ruette, N., et al. 2015, Phys. Rev. A, 92, 050702(R), doi: 10.1103/PhysRevA.92.050702

Habart, E., Boulanger, F., Verstraete, L., et al. 2003, Astron. Astrophys., 397, 623, doi: 10.1051/0004-6361:20021489

Habart, E., Boulanger, F., Verstraete, L., Walmsley, C., \& Des Forêts, G. P. 2004, Astron. Astrophys., 414, 531, doi: 10.1051/0004-6361:20031659

Hirama, M., Tokosumi, T., Ishida, T., \& Aihara, J.-i. 2004, Chem. Phys., 305, 307, doi: 10.1016/j.chemphys.2004.07.010

Hollenbach, D., \& Salpeter, E. E. 1971, Astrophys. J., 163, 155, doi: 10.1086/150754

Jochims, H. W., Baumgrtel, H., \& Leach, S. 1999, Astrophys. J., 512, 500, doi: 10.1086/306752
Klærke, B., Toker, Y., Rahbek, D. B., Hornekær, L., \& Andersen, L. H. 2013, Astron. Astrophys., 549, A84, doi: 10.1051/0004-6361/201219952

Lifshitz, C. 2002, Eur. J. Mass Spectrom., 8, 85, doi: 10.1255/ejms.476

Ling, Y., Gotkis, Y., \& Lifshitz, C. 1995, Eur. Mass Spectrom., 1, 41, doi: 10.1255/ejms.158

Mackie, C. J., Candian, A., Huang, X., et al. 2018, Phys. Chem. Chem. Phys., 20, 1189, doi: 10.1039/C7CP06546A

Mayer, P. M., Blanchet, V., \& Joblin, C. 2011, J. Chem. Phys., 134, 244312, doi: 10.1063/1.3604933

Menk, R. H., Antonelli, M., Brajnik, G., et al. 2019, AIP Conf. Proc., 2054, 060071, doi: 10.1063/1.5084702

Mennella, V., Hornekær, L., Thrower, J., \& Accolla, M. 2012, Astrophys. J. Lett., 745, L2, doi: 10.1088/2041-8205/745/1/L2

Micelotta, E. R., Jones, A. P., \& Tielens, A. G. G. M. 2010, Astron. Astrophys., 510, A36, doi: 10.1051/0004-6361/200911682

Mishra, P. M., Avaldi, L., Bolognesi, P., et al. 2014, J. Phys. Chem. A, 118, 3128

Montillaud, J., Joblin, C., \& Toublanc, D. 2013, Astron. Astrophys., 552, A15, doi: 10.1051/0004-6361/201220757

Pauzat, F., \& Ellinger, Y. 2001, Mon. Not. R. Astron. Soc., 324, 355, doi: 10.1046/j.1365-8711.2001.04277.x

Plekan, O., Coreno, M., Feyer, V., et al. 2008, Phys. Scr., 78, 058105, doi: 10.1088/0031-8949/78/05/058105

Postma, J., Bari, S., Hoekstra, R., Tielens, A. G. G. M., \& Schlathölter, T. 2010, Astrophys. J., 708, 435

Rapacioli, M., Cazaux, S., Foley, N., et al. 2018, Phys. Chem. Chem. Phys., 20, 22427, doi: 10.1039/C8CP03024C

Rauls, E., \& Hornekær, L. 2008, Astrophys. J., 679, 531, doi: $10.1086 / 587614$

Reitsma, G., Boschman, L., Deuzeman, M. J., et al. 2014, Phys. Rev. Lett., 113, 53002, doi: 10.1103/PhysRevLett.113.053002

Rouillé, G., Krasnokutski, S. A., Fulvio, D., et al. 2015, Astrophys. J., 810, 114, doi: $10.1088 / 0004-637 x / 810 / 2 / 114$

Sandford, S. A., Bernstein, M. P., \& Materese, C. K. 2013, Astrophys. J. Suppl. Ser., 205, 8, doi: 10.1088/0067-0049/205/1/8

Schlathölter, T., Mostafa, Y., Kamman, A., et al. 2020, Eur. Phys. J. D, 74, 114, doi: 10.1140/epjd/e2020-10111-y

Solano, E. A., \& Mayer, P. M. 2015, J. Chem. Phys., 143, 104305, doi: 10.1063/1.4930000 
Stockett, M. H., Gatchell, M., Chen, T., et al. 2015, J.

Phys. Chem. Lett., 6, 4504, doi: 10.1021/acs.jpclett.5b02080

Szczepanski, J., Oomens, J., Steill, J. D., \& Vala, M. T. 2010, Astrophys. J., 727, 12, doi: 10.1088/0004-637X/727/1/12

Thrower, J. D., Friis, E. E., Skov, A. L., Jørgensen, B., \& Hornekær, L. 2014, Phys. Chem. Chem. Phys., 16, 3381, doi: 10.1039/C3CP54073A

Thrower, J. D., Jørgensen, B., Friis, E. E., et al. 2012, Astrophys. J., 752, 3, doi: 10.1088/0004-637X/752/1/3

Tielens, A. G. G. M. 2005, The Physics and Chemistry of the Interstellar Medium (Cambridge University Press). http://dx.doi.org/10.1017/CBO9780511819056

—. 2008, Annu. Rev. Astron. Astrophys., 46, 289, doi: 10.1146/annurev.astro.46.060407.145211
Vala, M., Szczepanski, J., Oomens, J., \& Steill, J. D. 2009, J. Am. Chem. Soc., 131, 5784, doi: 10.1021/ja808965x

Wagner, D. R., Kim, H., \& Saykally, R. J. 2000, Astrophys. J., 545, 854, doi: 10.1086/317868

West, B., Joblin, C., Blanchet, V., et al. 2014a, J. Phys. Chem. A, 118, 1807, doi: 10.1021/jp500430g

West, B., Lowe, B., \& Mayer, P. M. 2018, J. Phys. Chem. A, 122, 4730, doi: 10.1021/acs.jpca.8b02667

West, B., Sit, A., Mohamed, S., et al. 2014b, J. Phys. Chem. A, 118, 9870, doi: 10.1021/jp505438f

West, B., Useli-Bacchitta, F., Sabbah, H., et al. 2014c, J. Phys. Chem. A, 118, 7824, doi: 10.1021/jp506420u

Wolf, M., Giacomozzi, L., Gatchell, M., et al. 2016a, Eur. Phys. J. D, 70, 1, doi: 10.1140/epjd/e2016-60735-3

Wolf, M., Kiefer, H. V., Langeland, J., et al. 2016b, Astrophys. J., 832, 24. http://stacks.iop.org/0004-637X/832/i=1/a=24 